\title{
Evaluación y certificación de competencias laborales en México El caso de las dependencias del gobierno federal
}

\author{
Lourdes Álvarez Medina * \\ Claudia De la O Pérez **
}

\section{Resumen}

En este artículo se analiza el proceso de certificación de competencias laborales en cinco dependencias del gobierno federal. Nos interesa definir los factores que inciden en la actividad de certificación: qué normas se certifican, cómo se eligen, cuáles son las experiencias y aprendizaje en el proceso de evaluación y cómo se circunscribe la certificación en su nuevo sistema de administración del capital humano. Se examinan estadísticas; se estudian cinco casos de certificación y se analiza la relación entre certificación y la nueva Ley del Servicio Profesional de Carrera. Se concluye que las dependencias del gobierno son las organizaciones que mayor actividad han tenido en el proceso de certificación, el cual ha sido impulsado por el poder ejecutivo. Se han certificado principalmente en tres normas que han sido seleccionadas por dos razones: formar cuadros de capacitadores y evaluadores para capacitarse y certificarse ellos mismos y la selección de normas transversales que se pueden aplicar al personal de diferentes áreas y niveles jerárquicos de la empresa. Dentro de las experiencias de evaluación se observa la fragilidad del entorno y el aprendizaje del evaluador para enfrentar las situaciones que afectan su actuación.

Palabras clave: certificación, evaluación, competencias laborales, gobierno, México.

\footnotetext{
* Investigadora de la División de Investigación de la Facultad de Contaduría y Administración de la UNAM. Correro electrónico: lourdes3055@yahoo.com

** Evaluadora de Competencias Laborales y alumna de la División de Estudios de Posgrado de la FCA-

UNAM. Correro electrónico: delaosito@yahoo.com
} 


\section{Introducción}

En el marco de las negociaciones del Tratado de Libre Comercio de América Cdel Norte (TLCAN) se decidió implantar el sistema de educación y certificación basado en normas de competencia laboral en México.

La formación basada en competencia laboral proviene de una corriente de pensamiento social contemporáneo que pone énfasis en la vinculación entre el sistema educativo y el productivo para lograr la afinidad entre la capacitación de la mano de obra y las necesidades de las empresas. ${ }^{1}$

El sistema inició en el Reino Unido en la década de los ochenta; después se implementó en países como Australia, Nueva Zelanda, Francia, Alemania, Estados Unidos y Canadá.Al adoptar el sistema se esperaba que la competitividad de la mano de obra mejorara; sin embargo, los estudios realizados no han sido suficientes para saber si se han alcanzado estos objetivos. ${ }^{2}$

Definir el concepto de competencia ha sido difícil porque es un constructo que se deduce del desempeño de los individuos y deriva de su dominio de conocimientos, valores, destrezas y actitudes necesarias para la realización de un trabajo efectivo. ${ }^{3}$

Los supuestos del modelo de evaluación basada en competencias señalan que: a) es posible establecer normas de competencia laboral públicas, b) un gran número de aprendices puede alcanzarlas, c) las normas pueden reflejar diferentes desempeños, d) los evaluadores pueden internalizar las normas y e) los evaluadores pueden juzgar diferentes desempeños de manera sistemática. ${ }^{4} \mathrm{Si}$ bien se han realizado diversos estudios para analizar los resultados del sistema y los problemas de evaluación, se ha puesto poca atención al proceso de aprendizaje del evaluador:

\footnotetext{
${ }^{1}$ Leonard Mertens, Competencia laboral: sistemas, surgimientos y modelos, Organización Mundial del Trabajo, Montevideo, 1996, p. V.

${ }^{2}$ Deborah Kellie, "The australian way: competency based training in the corporate sector", International Journal of training and development, vol 3, no. 2, p. 118, jun, 1999; A. Gonczi y P. Hager, "Competency based standars: A boom for continuing professional education", en Studies in Continuing Education, vol. 13 , no. 1 , pp. $24-40$.

3 Ibid.

${ }^{4}$ Catherine Taylor, "Assessment for measurement of standards: the peril and promise of large scale assessment reform", en American Educational Research Journal, vol. 31, no. 2, 1994, pp. 231-262.
} 
cuáles son sus experiencias de evaluación, cómo se organiza para llevarla a cabo, cómo aprende a juzgar diferentes desempeños de manera sistemática y cómo aprende a enfrentar situaciones inesperadas o cambiantes que afectan su práctica.

El sistema de competencias laborales en México se ha desarrollado en dos vertientes: la educativa y la laboral. En este trabajo abordamos la certificación en el sector laboral, específicamente se analiza el proceso de certificación de competencias laborales en las dependencias del gobierno federal. Nos interesa definir los factores que inciden en la actividad de certificación: qué normas se certifican, cómo se seleccionan, cuáles son las experiencias y aprendizaje en el proceso de evaluación y certificación para el evaluador y cómo se relaciona la certificación de competencias laborales con el sistema de administración del capital humano.

Esta investigación se basa en fuentes primarias y secundarias: en primer lugar se analizan las estadísticas generales del sistema de certificación; después se estudian cinco casos de certificación de competencia laboral en dependencias del gobierno federal describiendo el proceso y experiencias de evaluación; finalmente, se analizan las disposiciones que contiene la nueva Ley del Servicio Profesional de Carrera con respecto a la certificación y administración de capital humano del gobierno federal que entra en vigor en enero de 2005. Se utilizan entrevistas, reportes de CONOCER, de la Secretaría de Educación Pública, de la Secretaría de la Función Pública, del Banco Interamericano de Desarrollo, libros y revistas especializadas.

\section{Antecedentes de la certificación basada en normas de competencia laboral}

Por competencia laboral se entiende la capacidad productiva de un individuo que se define y mide en términos de desempeño en un determinado contexto laboral; contempla los conocimientos, habilidades, destrezas y actitudes necesarias para la realización de un trabajo efectivo y calidad. ${ }^{5}$

A mediados de la década de los ochenta, el sistema de educación, capacitación y certificación basado en normas de competencia laboral fue implantado en el

${ }^{5}$ L. Mertens, op. cit., p. V. 
Reino Unido; desde entonces ha mutado y se ha establecido en varios países. Los motivos para adoptar el sistema fueron económicos, por lo que el impulso para la implantación ha venido de los gobiernos de los diferentes países debido a que se consideró que los educadores tradicionales no respondían adecuadamente a las necesidades del sector industrial. ${ }^{6}$

Los educadores han criticado este sistema por promover una visión de la educación y capacitación basada en principios conductistas; no se consideran adecuadamente los conocimientos, los valores y las actitudes esenciales para la adquisición de habilidades y conocimientos técnicos. Se señala que las normas de competencia se relacionan con el logro de objetivos industriales de corto plazo y no con la adquisición de habilidades o de la educación del individuo. ${ }^{7}$ La educación basada en normas de competencia tiene una visión prescriptiva, determinada por normas industriales; no se interesa en determinar los motivos por los que debe llevarse a cabo una tarea o en cómo aplicar este conocimiento a otras situaciones.

Por otra parte, ha habido reformadores educativos que consideran este adiestramiento como una forma de proporcionar educación tanto general como vocacional; asimismo, señalan que mientras el curriculum tradicional privilegia el conocimiento abstracto, este sistema permite al estudiante saber exactamente qué es lo que se requiere que aprenda y cómo relacionarlo con la práctica. ${ }^{8}$

Uno de los temas más polémicos de la instrumentación del sistema ha sido la evaluación de las competencias que inicialmente se basaban en enfoques de carácter conductista. Al evaluar se observaba a la persona en el proceso de realizar una tarea concreta y se comparaba con una especificación detallada de la tarea que había sido normalizada por un grupo de expertos. Los cambios, que pretenden una nueva noción de validez, implican la recopilación de pruebas o evidencias de desempeño y la aplicación de juicios profesionales. ${ }^{9}$

${ }^{6}$ J. S. Dawkins, Skills for Australia, Minister for employment, Education and Training, Canberra, 1987, p. 16.

7 Nancy Jackson, "Reforming vocational learning: Contradictions of competency", en What future for technical and vocational education and training, vol. 2, NCVER, Adelaide, 1992, pp. 187-200.

${ }^{8}$ Andrew Gonczi, "Enfoques de la educación basada en competencias: la experiencia australiana", La academia, nov-dic, 1997, p.1 en http://www.hemerodigital.unam.mx/ANUIES/ipn/academia/12/sec_9.htm

${ }^{9}$ Ibid., pp. 5, 10 y 11. 
También se han estudiado los problemas que se presentan al realizar las evaluaciones en diferentes contextos; no es lo mismo realizar una evaluación en instituciones formales de educación a realizarla en un contexto de trabajo. De igual forma, se ha discutido la separación de funciones y las atribuciones entre los evaluadores que se encuentran en el centro de trabajo y los educadores que laboran en las instituciones formales. ${ }^{10}$ Sin embargo, se sabe muy poco sobre la actuación del evaluador y el proceso de aprender a evaluar; por ejemplo, cuáles son sus experiencias durante el proceso de evaluación, cómo aprende a aplicar las pruebas y a organizarse, cómo aprende a juzgar diferentes desempeños de manera sistemática. Es evidente que debe haber más estudios y un mayor debate respecto al proceso de evaluación porque de éste dependen en parte los resultados positivos que pueda tener el sistema.

\section{Resultados generales del sistema de evaluación y certificación en México}

El sistema de evaluación y certificación de competencias laborales en México inició a finales de 1993 mediante un proyecto sobre educación tecnológica y modernización de la capacitación que desarrollaron de manera conjunta la Secretaría de Educación Pública y la Secretaría del Trabajo y Previsión Social. Dos años después se creó el Consejo de Normalización y Certificación de la Competencia Laboral (CONOCER) que es la base para la operación del Proyecto de Modernización de la Educación Técnica y la Capacitación (PMETyC). ${ }^{11}$

Este proyecto que inició con un fondo de 30 millones de dólares aportados por el Banco Mundial pretende actuar sobre el sistema educativo y el laboral con planes y programas de estudio, basados en normas de competencia laboral en instituciones de educación técnica. Inicialmente el proyecto se limitó a instituciones terminales como el Colegio Nacional de Educación Profesional Técnica (Conalep), los Centros de Capacitación del Trabajo Industrial (Cecatis), y los Centros de Estudios Tecnológicos Industriales (Cetis). En la segunda fase del proyecto se incluyeron otras instituciones como las de educación superior que estaban interesadas.

\footnotetext{
${ }^{10}$ Andrew Gonczi, "Problemas asociados con la implantación de la educación basada en competencias: de lo atomístico a lo holístico", p.162 en Organización Internacional del Trabajo, Formación basada en competencia laboral, México, 1997, 262 pp.

11 Véase http://www.conocer.org.mx
} 
Las acciones de CONOCER para desarrollar el sistema desde el punto de vista laboral han contemplado las siguientes acciones:

a) Se creó el Sistema de Normalización de Competencia Laboral, que es responsable de elaborar las normas técnicas ${ }^{12}$ que deberán utilizarse para evaluar la competencia laboral de un individuo.

b) Se establecieron mecanismos para crear y operar el sistema de certificación. Se acreditaron organismos certificadores, que a su vez acreditan a centros de evaluación. Estos organismos son responsables también de elaborar los instrumentos de evaluación para cada norma que certifican para la emisión de certificados y para la formación de bases de datos para el control.

En México, la certificación no es obligatoria, pero se empiezan a desarrollar mecanismos para transformar la oferta de formación y capacitación, así como para estimular la certificación de competencias; algunas empresas se están organizando por competencias y seleccionando personal certificado.

En 2003, el Banco Mundial le retiró el apoyo a CONOCER porque los resultados de la gestión no correspondieron a la propuesta presentada, además, hubo un reporte de incumplimientos. ${ }^{13}$ El sistema se paralizó por un tiempo y entró en una etapa de redefinición de estructura jurídica. De acuerdo con el último comunicado, la Secretaría de Educación Pública será la responsable del PMETyC y concentrará la administración de la educación, capacitación y normalización para lo cual contará con un crédito del Banco Interamericano de Desarrollo (BID) por 204 millones de dólares. A este crédito se podrá acceder en los próximos seis años, siempre y cuando se cumplan las condiciones señaladas en el Programa Multifase de Formación de Recursos Humanos Basada en Competencias. ${ }^{14}$ El programa señala que promoverá la competencia laboral en las empresas mediante una estrategia de focalización en cuatro sectores de la economía: ${ }^{15}$

\footnotetext{
${ }^{12}$ Una norma describe los conocimientos, habilidades, destrezas y actitudes que una persona debe tener; cómo puede juzgarse si lo que hizo está bien hecho; bajo qué condiciones la persona tiene que demostrar su aptitud y los tipos de evidencias necesarios. Se considera transversal cuando puede ser aplicada en diversas áreas de la organización y diferentes niveles jerárquicos.

${ }_{13}^{13}$ Información aportada por un exfuncionario, líder de proyecto de CONOCER, 30 abril de 2004.

${ }^{14}$ BID, op. cit.

${ }^{15}$ Ibid, pp. 6-9.
} 
- Fabricación de aparatos domésticos. En este sector, la norma se enfocará a los servicios posventa; se estima certificar 9400 trabajadores en los próximos cuatro años.

- Minería. Este sector ya cuenta con 10 normas y se considera desarrollar cinco nuevas normas en las que se pretende certificar 9600 personas en actividades que incrementen seguridad y reduzcan accidentes laborales.

- Distribución de llantas. Se certificará en servicios de posventa, mantenimiento y reparación de llantas para lo que se pronostica certificar a 5000 personas en los próximos tres años.

- Turismo. Se busca reducir costos administrativos y operativos, así como mejorar la calidad de los servicios y piensan certificar a 7590 personas.

A continuación se presentan algunos resultados que nos permiten ubicar de manera general los resultados del sistema.

\section{Cuadro 1}

Indicadores del sistema de certificación y normalización de competencia laboral en México

\begin{tabular}{|rl|ll|l|}
\hline Año & $\begin{array}{l}\text { Creación de } \\
\text { nuevos } \\
\text { organismos } \\
\text { certificadores }\end{array}$ & $\begin{array}{l}\text { Creación de } \\
\text { nuevos centros } \\
\text { de evaluación }\end{array}$ & $\begin{array}{l}\text { Número de } \\
\text { normas } \\
\text { elaboradas }\end{array}$ & $\begin{array}{l}\text { Emisión de } \\
\text { certificados } \\
\text { por unidad } \\
\text { dictaminada }\end{array}$ \\
\hline & 7 & & & 364 \\
\hline 1998 & 5 & 158 & 150 & 5621 \\
\hline 1999 & 11 & 433 & 141 & 47396 \\
\hline 2000 & 7 & 212 & 72 & 46787 \\
\hline 2001 & 2 & 390 & 89 & 58000 \\
\hline 2002 & cero & 122 & 60 & 47738 \\
\hline 2003 & $\mathbf{3 2}$ & $\mathbf{1 3 2 4}$ & $\mathbf{6 6 7}$ & $\mathbf{2 0 5} \mathbf{9 0 6}$ \\
\hline Total & & & &
\end{tabular}

Fuente: Resumen de resultados de CONOCER 2002 y 2003

*Debido a que un mismo centro de evaluación se puede certificar dos veces, en realidad existen 1068 centros

\footnotetext{
${ }^{16}$ Una norma está compuesta de varias unidades. Se puede certificar a un individuo en todas las unidades de la norma o sólo en algunas.
} 
Inicialmente, el CONOCER realizó la promoción del sistema de certificación dando apoyo económico a los organismos certificadores ${ }^{17}$, por lo que se aprecia un rápido crecimiento de los cuatro indicadores hasta el año 2000 (ver cuadro 1). Después se observa una disminución en la creación del número de centros de evaluación y en el número de normas creadas. Sin embargo, la emisión de certificados siguió creciendo hasta el 2002 en que se alcanzaron 58 mil certificaciones anuales. En 2003 hubo una ligera disminución de la actividad que podría explicarse por falta de credibilidad del sistema, debido a que el Banco Mundial les retiró el apoyo y ha sido necesario redefinir las figuras jurídicas. ${ }^{18}$

Respecto a la elaboración de normas se observa que el número ha ido disminuyendo cada año, además de que su uso no es el esperado: de 667 normas elaboradas, únicamente 308 fueron adquiridas por los organismos certificadores; éstos a su vez otorgaron los derechos de 256 normas a los centros de evaluación, quienes mantienen en uso 170 normas. Tener en actividad solamente $28 \%$ de las normas es alarmante si se considera que en promedio cada norma ha costado 27 mil dólares. ${ }^{19}$

${ }^{17}$ Entrevista realizadas con el director de un centro de evaluación en marzo de 2004.

18 Ibid.

${ }^{19}$ Banco Interamericano de Desarrollo, Programa multifase de formación de recursos humanos con orientación de competencia, fase I, noviembre, 2003, p. 3. 


\section{Cuadro 2}

Participación de centros de evaluación en el total de unidades dictaminadas (1998-2003)

\begin{tabular}{|l|rr|r|r|}
\hline $\begin{array}{l}\text { Tipo de centro de } \\
\text { evaluación }\end{array}$ & $\begin{array}{l}\text { Total de } \\
\text { centros }\end{array}$ & $\begin{array}{l}\text { Unidades } \\
\text { dictaminadas }\end{array}$ & $\begin{array}{l}\text { \% de } \\
\text { unidades } \\
\text { por centro }\end{array}$ & $\begin{array}{l}\text { No. de unidades } \\
\text { dictaminadas en } \\
\text { promedio por } \\
\text { cada centro }\end{array}$ \\
\hline Escuelas públicas & 610 & 88539 & $\mathbf{4 3 \%}$ & 145.14 \\
\hline $\begin{array}{l}\text { Escuelas } \\
\text { privadas }\end{array}$ & 48 & 4118 & $2 \%$ & 85.79 \\
\hline Empresas privadas & 329 & 47358 & $23 \%$ & 143.94 \\
\hline $\begin{array}{l}\text { Empresas o } \\
\text { dependencias } \\
\text { públicas }\end{array}$ & 128 & 43240 & $21 \%$ & $\mathbf{3 3 7 . 8 1}$ \\
\hline $\begin{array}{l}\text { Cámaras } \\
\text { empresariales }\end{array}$ & & & & \\
\hline $\begin{array}{l}\text { Asociaciones } \\
\text { Evaluadores } \\
\text { independientes } \\
\text { Sindicatos }\end{array}$ & 48 & 6178 & $3 \%$ & \\
\hline Total & 46 & 16473 & $8 \%$ & 03.04 \\
\hline
\end{tabular}

Fuente: Elaboración propia con base en el resumen de resultados de CONOCER, 2002 y 2003

En el cuadro 2 se observa la dinámica de los centros de evaluación cuando se calcula el número de unidades dictaminadas en promedio por cada centro. En primer lugar se encuentran las empresas o dependencias públicas que tienen un promedio de 337 unidades dictaminadas por cada centro. Esta intensa actividad se explica por los acuerdos establecidos sobre el servicio profesional de carrera, donde se señala la obligación de la profesionalización de la educación y capacitación en el trabajo en materia de competencia laboral en instituciones gubernamentales. ${ }^{20}$

En segundo lugar se observan las asociaciones que tienen en promedio 257 unidades certificadas por centro, a pesar de que únicamente han participado 64 asociaciones que representan el $4.8 \%$ del total.

${ }^{20}$ Diario Oficial de la Federación, “Ley del Servicio Profesional de Carrera”, 10 de abril de 2003. 
Las escuelas públicas y las empresas privadas se encuentran en tercero y cuarto lugares. Tienen una dinámica muy parecida porque han certificado en promedio 145 y 143 unidades por centro, respectivamente. Por otro lado, si consideramos el tamaño, las escuelas públicas son las que cuentan con más centros de evaluación — 610 que representan el $46 \%$ de los centros - y han certificado el $43 \%$ de las unidades.

En el cuadro 3 se presentan las unidades dictaminadas por área y nivel de competencia. Se observan tres áreas que han concentrado el 72\% de las unidades certificadas. El área de Servicios de Finanzas, Gestión y Soporte Administrativo ha certificado 74,026 unidades, lo que corresponde al 36\% del total; de éstas la mayor parte se han certificado en el segundo nivel. ${ }^{21}$ En segundo lugar se encuentra el área de Ventas de bienes y servicios que ha certificado 39,388 unidades, lo que corresponde al 19\% del total; cabe resaltar que esta área es la única que ha dictaminado unidades en el quinto nivel de competencia y que son principalmente las tiendas departamentales quienes han tenido gran actividad. En tercer lugar tenemos el área de Manufactura con un total de 34,848 unidades certificadas, en donde la industria textil ha tenido una participación importante certificando $76 \%$ de las unidades en la norma de "vestido".

\section{Cuadro 3}

\section{Unidades dictaminadas por área y nivel de competencia}

\begin{tabular}{|c|c|c|c|c|c|c|c|c|c|c|c|c|c|}
\hline Área & 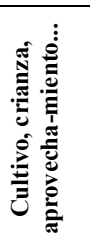 & 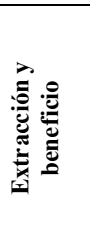 & 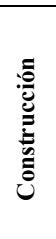 & 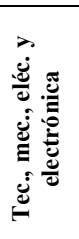 & 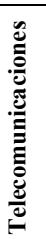 & 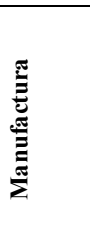 & 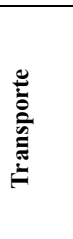 & 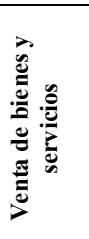 & 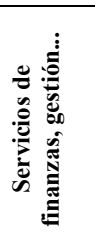 & 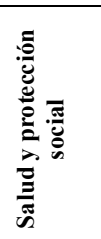 & 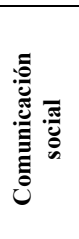 & 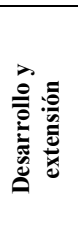 & 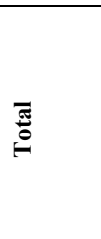 \\
\hline 5 & & & & & & & & 5,530 & & & & & 5,530 \\
\hline 4 & & & & 18 & & 48 & & 171 & 3,390 & & & 4 & 3,631 \\
\hline 3 & & & 77 & & 39 & 2,741 & 482 & 9,950 & 325 & 586 & & 5,924 & 20,124 \\
\hline 2 & 10,123 & 7,490 & 418 & 5,430 & & 5,615 & 5,763 & 21,510 & 70,311 & 9,550 & 3,002 & & 139,212 \\
\hline 1 & 4,539 & 3,180 & & & & 26,444 & 1,019 & 2,227 & & & & & 37,409 \\
\hline Total & 14,662 & 10,670 & 495 & 5,448 & 39 & 34,848 & 7,264 & 39,388 & 74,026 & 10,136 & 3,002 & 5,928 & 205,906 \\
\hline
\end{tabular}

Fuente: Resumen de resultados de CONOCER, julio de 2003

\footnotetext{
${ }^{21}$ Los cinco niveles de competencia de la norma describen grados de dificultad en una competencia. El primer nivel evalúa una competencia en una variada gama de actividades laborales en su mayoría rutinarias y predecibles; el quinto nivel implica la aplicación de una importante gama de principios y técnicas complejas en una amplia e impredecible variedad de contextos y con un grado considerable de autonomía y responsabilidad por el trabajo de otros y por la distribución de recursos sustanciales.
} 
El área de Servicios de Finanzas, Gestión y Soporte Administrativo tiene cuatro grupos que indican cuáles son las normas más certificadas; a continuación los detallamos:

a) Servicios de informática (nivel 2) con 65,867 unidades dictaminadas. En esta área la norma certificada es la de Elaboración de Documentos Mediante Herramientas de Cómputo.

b) Trabajo de oficina (nivel 2) con 4,186 unidades dictaminadas. En esta área la norma certificada es la de Atención a Clientes Mediante Información Documental.

c) Administración de recursos humanos con 2, 895 unidades dictaminadas. En esta área la norma certificada es la de Diseño e Impartición de Cursos de Capacitación.

d) Comercio, servicios educativos, administración pública y servicios aduanales con 988 unidades dictaminadas.

De acuerdo con la información y las tres tablas analizadas, en este punto se puede concluir que las dependencias del gobierno son las organizaciones que mayor actividad han tenido en el proceso de certificación, que éste ha sido impulsado por el poder ejecutivo y la norma que más han certificado es la de Elaboración de Documentos Mediante Herramientas de Cómputo.

\section{Casos de evaluación de competencia laboral en dependencias guberna- mentales}

A continuación se presenta un resumen de los cinco casos que permiten analizar las circunstancias y experiencias de evaluación. ${ }^{22}$

\footnotetext{
${ }^{22}$ Se entrevistó a la Lic. Claudia De la O quien fue la evaluadora de los 256 empleados en las cinco dependencias del gobierno federal. En primer lugar se le pidió escribir sus experiencias de evaluación y a partir de ese documento se realizaron cuatro entrevistas de acuerdo con la metodología de Francisco Sierra, "Función y sentido de la entrevista cualitativa en la investigación social", en Jesús Galindo (coord.), Técnicas de investigación en sociedad, cultura y comunicación, México, Pearson Education, 1998, pp. 276-339.
} 
Tabla 5

\section{Información sobre las dependencias del gobierno federal que fueron certificadas}

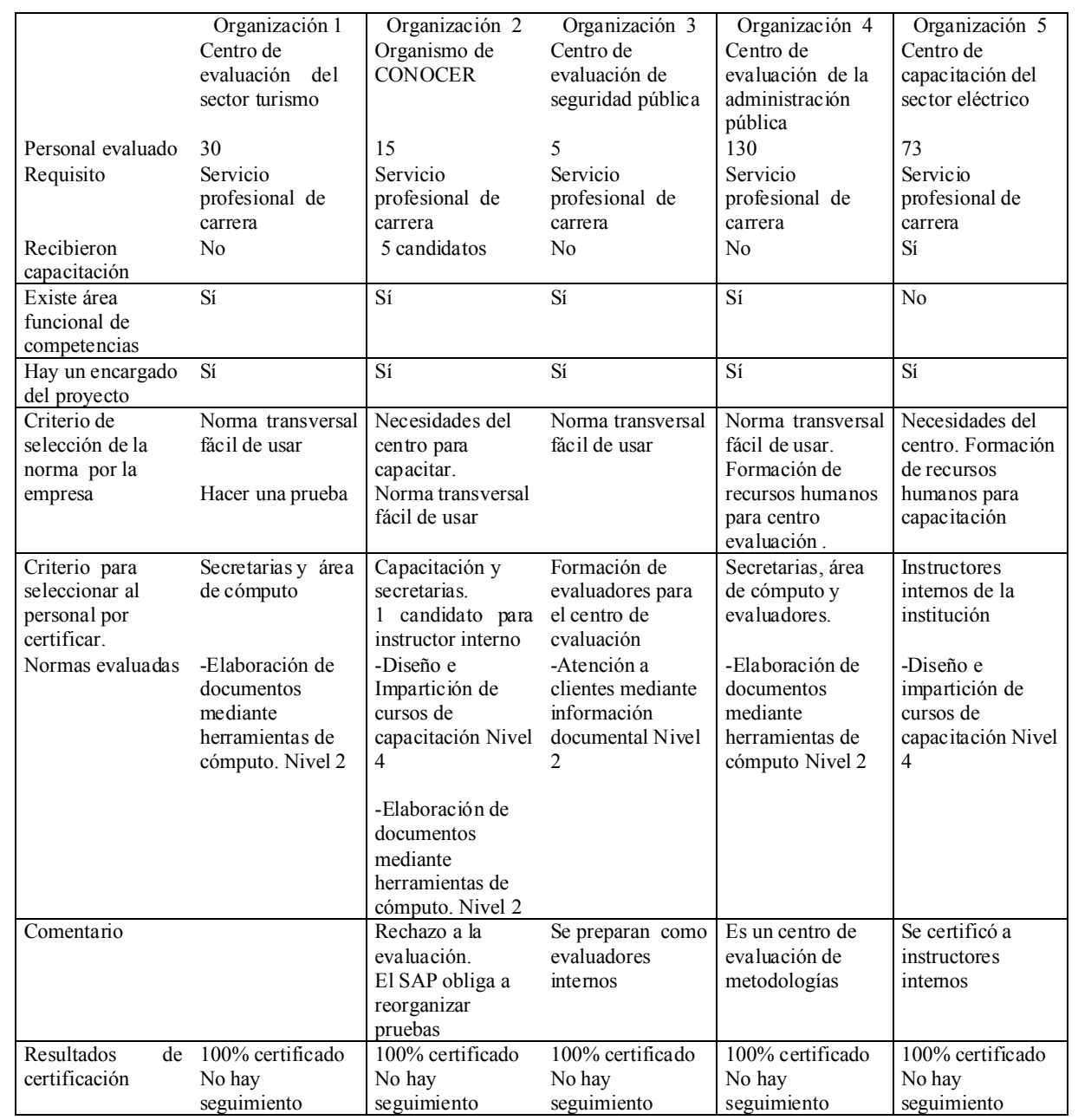

${ }^{23}$ Software denominado SAP, que cubría con algunas de las evidencias porque automáticamente elabora reportes que se le piden al empleado que elabore; por lo tanto, se programaron pruebas específicas. 


\section{Empresa 1. Sector turismo, certificada en 2001}

Se inició un proyecto para certificar a 30 miembros de la organización, la cual funge como centro de evaluación. Se certificó a 30 integrantes del cuerpo de evaluadores y verificadores internos de la organización. Se atendieron las delegaciones regionales de Baja California, Chiapas, Puebla, Sonora, Tamaulipas, Veracruz, Quintana Roo y Distrito Federal, en la primera fase; en la segunda fase las delegaciones regionales de Sonora, Veracruz y Distrito Federal.

\section{Empresa 2. Organismo de CONOCER, certificada en 2002}

Se inició un proyecto para certificar a 15 miembros de la institución, la cual participa en el Sistema de Normalización y Certificación de la Competencia Laboral. Uno de los candidatos fungiría como instructor interno de los procedimientos metodológicos del sistema. Debido a que fue el primer proyecto de la organización, se apreció cierto rechazo a la evaluación por parte del personal ubicado en mandos medios; también se detectaron limitaciones en algunas tareas de las funciones relativas a la norma, pues cuentan con un software denominado SAP que cubría algunas de las evidencias, para poder evaluar se programó capacitación y pruebas específicas.

\section{Empresa 3. Seguridad Pública, certificada en 2002-2003}

Se inició un proyecto para certificar a 5 miembros de la organización, que se están preparando como evaluadores del centro de evaluación interno. Después de certificarlos se presentó una nueva propuesta para la certificación de los 5 candidatos en otra norma. Este proyecto no se realizó porque se dieron cambios de personal y de estructura organizacional. En el primer proyecto se mantuvo una buena relación con el subdirector de área, quien fungía como responsable del proyecto, pero se perdió la posibilidad de hacer el seguimiento porque fue transferido. Posteriormente, se indicó que no tenían apoyo económico para los proyectos de certificación y se suspendieron las relaciones.

\section{Empresa 4. Sector Administración de la Función Pública, certificada en 2003-2004}

Se inició un proyecto para certificar a 130 miembros de la organización, quienes participan como colaboradores de la institución que está constituida como centro de evaluación de metodologías. Se mantuvo una buena relación con la coordinadora del centro de evaluación; no hubo dificultades porque la dependencia tiene experiencia en certificación. 


\section{Empresa 5. Sector Eléctrico 2003-2004}

Se inició un proyecto para certificar a 73 miembros de la organización, quienes participan como instructores internos de la institución en diferentes regiones como son: Puebla, Querétaro, Guerrero, Hidalgo, Texcoco, y Cuernavaca. Actualmente, se encuentra abierto el programa de evaluación. Se mantuvo una buena relación con el líder del proyecto del área central; no hubo contratiempos debido a que la organización se encuentra involucrada y sensibilizada en el sistema de certificación.

Con lo anterior se buscaba conocer el proceso de evaluación que se lleva en la práctica, los problemas que se van encontrando en la aplicación de la evaluación, el aprendizaje del evaluador y cómo se aprende a juzgar diferentes desempeños de manera sistemática.

\subsection{Problemas detectados en el campo de aplicación}

En resumen se podría decir que hay problemas de comunicación, confianza, ética y procedimientos estandarizados.

- Los factores que el evaluador relaciona con la satisfacción del cliente son: cumplimiento con el programa inicial de evaluación; la obtención de juicio de competencia laboral para el $100 \%$ de los candidatos programados; adecuación a las necesidades del cliente, como la adaptación en el número de personas evaluadas o tiempo requerido; presentación del informe final claro y con evaluación del servicio prestado, observando puntualidad, instrucciones claras al candidato, aclaración de las dudas, ambiente de confianza y respeto, información oportuna de los resultados de evaluación al candidato.

- El evaluador aprende en la práctica al enfrentar situaciones inesperadas que tiene que resolver; por ejemplo, comunicarle al evaluado que el examen se suspende porque le faltan conocimientos, detectar a las personas que sabotean la evaluación para que no les asignen más responsabilidades, detectar y reportar a las personas que hacen trampa tratando de conseguir las pruebas que ya fueron aplicadas a sus compañeros.

- Las condiciones para trabajar son diferentes en cada organización. Se pueden tener espacios especiales para evaluar, pero en ocasiones hay que hacer la evaluación en el lugar de trabajo del empleado; esto ocasiona interrupciones y demoras de varias horas en la evaluación. 


\subsection{Problemas con respecto a la comunicación con los candidatos}

- Después de aplicar varias pruebas, el evaluador alcanzó a comprender que hay un gran nerviosismo en el empleado. Para tranquilizarlo y lograr mayor concentración empezó a aplicar técnicas de programación neurolinguística; en éstas el evaluador pone énfasis en dar confianza y respeto al personal evaluado.

- Los candidatos temen que en el diagnóstico se identifiquen fallas que afectarán posibles promociones o permanencia en sus puestos y también les preocupa que se les obligue a hacer más trabajo. Estas situaciones responden a la dinámica interna de la organización y a la mala comunicación por parte de los responsables de los programas de evaluación de competencia laboral de la institución.

- Personal seleccionado para ser evaluado: para la certificación en la norma Elaboración de Documentos Mediante Herramienta de Cómputo las organizaciones seleccionaron a secretarias ejecutivas o personal del área de cómputo; sin embargo, en algunas ocasiones se incluyeron subdirectores. Esto ocasionó problemas porque argumentaron que no era el nivel de norma requerido para ellos, pero aceptaron evaluarse.

\subsection{Problemas con respecto a las evidencias}

- El evaluador forma situaciones artificiales para poder evaluar, lo que ocasiona que las actitudes se evalúen de manera relativa.

- La norma de herramientas de cómputo no contempla las funciones del SAP, debido a esto fue necesario hacer algunas adaptaciones de las pruebas.

- No se hace una evaluación de desempeño ni antes ni después de la certificación, por lo que no se pueden comprobar los beneficios de la misma, ni se puede comprobar si la certificación aporta algo a la organización. Las empresas se muestran renuentes a este proceso; cuando se sugiere la evaluación hay un ligero rechazo tanto de los encargados de la certificación en la empresa como de los evaluadores externos.

\subsection{Problemas con respecto al funcionamiento del sistema}

- Las dependencias están formando cuadros de capacitadores y evaluadores para después certificarse ellos mismos. Esta situación no debería permitirse porque el sistema pierde credibilidad. 
- La dinámica de la certificación también es afectada por la necesidad de gastar el presupuesto antes de que termine el año. Las instituciones buscan utilizar los remanentes del presupuesto del año, por lo que en esta temporada hay más solicitudes de las empresas para evaluar. Esto ocasiona que se contrate a evaluadores no certificados en la norma que van a evaluar, lo que no está permitido; sin embargo, los organismos certificadores no han hecho hincapié en que se respete este requisito porque no hay suficientes evaluadores.

- Ha habido mucha confusión en la puesta en marcha del sistema y hay variaciones significativas en la forma en que se proporciona el servicio; por ejemplo, el tiempo en que evalúan una misma norma y el monto que cobran por la evaluación. Esto lleva a prácticas de inteligencia en que los centros de evaluación tratan de conocer los métodos de trabajo de la competencia y contratar al personal con experiencia en certificación.

- El evaluador aprende a poner condiciones en las propuestas de trabajo; por ejemplo, si se va a evaluar una norma en 10 horas, se pide tiempo adicional para nivelar la pérdida de tiempo que ocasionan las distracciones del empleado por su trabajo, además de aprender; si un candidato no tiene el perfil sugerido no se le evalúa. Es necesario incluir todos estos puntos en las propuestas de trabajo porque representan costos extras para el centro y distracciones para el evaluador que es quien tiene el contacto personal con los candidatos.

\section{Relación de la certificación de competencias laborales con el sistema de administración de capital humano}

La Ley del Servicio Profesional de Carrera en la administración pública federal establece las bases para organizar el sistema de servicio profesional de carrera. En éste es obligatorio certificarse para desempeñar un cargo y no se permiten servidores públicos de libre contratación o asignación, como es el gabinete de apoyo de los secretarios, ni trabajadores de base. ${ }^{24}$ A continuación se presentan los aspectos relevantes en la organización del sistema de servicio profesional de carrera.

\footnotetext{
${ }^{24}$ Para una explicación parcial del nuevo sistema de servicio profesional de carrera consultar: http:// formacion.innova.gob. $\mathrm{mx} /$
} 
El sistema de administración de capital humano está formado por siete subsistemas: planeación de recursos humanos, ingreso, desarrollo profesional, capacitación y certificación de capacidades, evaluación de desempeño, separación y control y evaluación. ${ }^{25}$ Así se establece la creación de un registro o padrón con información del personal y su actuación, que es el enlace entre los siete subsistemas. Con esto se pretende tener un control adecuado del personal: quiénes son, dónde están, cómo se desempeñan y a dónde van.

El desarrollo profesional será el proceso mediante el cual los servidores públicos de carrera, con base en el desempeño, podrán tener movilidad en cualquier dependencia o en las entidades públicas y en las instituciones con las cuales exista convenio para tal propósito. ${ }^{26}$ Las dependencias podrán celebrar convenios con autoridades federales, estatales, municipales y del Distrito Federal. Asimismo, podrán negociar con organismos públicos o privados para el intercambio de recursos humanos, una vez cubiertos los perfiles requeridos. ${ }^{27}$

La capacitación y la certificación de capacidades serán los procesos mediante los cuales los servidores públicos de carrera serán inducidos, preparados, actualizados y certificados para desempeñar un cargo en la administración pública. ${ }^{28} \mathrm{Se}$ tendrán como objetivos prepararlos para funciones de mayor responsabilidad o de naturaleza diversa y certificarlos en las capacidades profesionales adquiridas. ${ }^{29}$

Los servidores profesionales de carrera deberán ser sometidos a una evaluación para certificar sus capacidades profesionales cada cinco años. Las evaluaciones deberán acreditar que el servidor público ha desarrollado y mantenido, actualizado el perfil y aptitudes requeridos para el desempeño de su cargo. Esta certificación será requisito indispensable para la permanencia de un servidor público en el sistema y en su cargo. ${ }^{30}$ De no aprobar la evaluación se procederá a su separación de la administración pública federal.

\footnotetext{
${ }^{25}$ Secretaría de la Contraloría y Desarrollo Administrativo, "Ley del Servicio Profesional de Carrera", en Diario Oficial de la Federación, 10 de abril de 2003, 1era sección, Título primero, capítulo primero, artículo 13, p. 46.

${ }^{26}$ Ibid., arts. 35 y 36

27 Ibid., art. 43

${ }^{28}$ Ibid., art. 44

${ }^{29} \mathrm{Ibid}$., arts. 46 y 47

${ }^{30}$ Ibid., art. 52
} 
Se puede decir que el sistema integra la certificación de competencias laborales de una forma poco clara; por ejemplo, el proceso de ingreso señala que en la selección donde se incluyen exámenes de conocimientos y habilidades ${ }^{31}$ es el procedimiento que permite analizar la capacidad, conocimientos, habilidades y experiencias de los aspirantes, pero no indica la necesidad de estar certificado en normas específicas con el acuerdo con el puesto. Además, las normas de competencia contemplan los conocimientos, habilidades, destrezas y actitudes necesarias para la realización de un trabajo efectivo y de calidad ${ }^{32}$ y en la evaluación del sistema no se incluyen dichas actitudes.

\section{Conclusiones}

Las dependencias del gobierno federal son las organizaciones que mayor actividad han tenido en el proceso de certificación; éste ha sido impulsada por el poder ejecutivo y se ha trabajado principalmente sobre las normas de Elaboración de Documentos Mediante Herramientas de Cómputo y Diseño e Impartición de Cursos de Capacitación. La selección de normas para certificar las dependencias del gobierno se relaciona con dos factores: la formación de cuadros de capacitadores y evaluadores para las mismas dependencias y el uso de normas transversales que se pueden aplicar en el personal de diferentes áreas y niveles jerárquicos de la empresa.

La autoevaluación se observa como un problema; las organizaciones pueden preparar a su personal de capacitación, pero al crear su centro de evaluación y evaluarse a sí mismos hacen que se pierda credibilidad.

Las normas transversales son normas que pueden aplicarse en diferentes niveles jerárquicos y áreas funcionales, pero difícilmente la empresa mejorará sus capacidades para competir al enfocarse en este tipo de normas. Las empresas o dependencias gubernamentales deben establecer procedimientos para detectar sus competencias que sean realmente importantes para mejorar el desempeño de su función básica y con base en éstas seleccionar las normas que deseen certificar.

31 Ibid., art, 29

32 Ibid. 
Hay que establecer procedimientos para detectar cuáles actividades deben normalizarse en lugar de crear normas por acuerdo, puesto que esto no da resultado. El sistema de normalización debe ser impulsado por las necesidades de las empresas.

Con respecto a las experiencias de aprendizaje en el proceso de evaluación encontramos que el evaluador siente presión para acreditar como competente al evaluado. El evaluador señaló que uno de los factores que se pueden relacionar con la satisfacción del cliente es la acreditación de competencia laboral para todos los candidatos programados; esto quiere decir que si no se obtiene la certificación del cien por ciento de los evaluados el cliente estará insatisfecho. Por otro lado, es importante señalar lo difícil que resulta cuando un evaluador interno evalúa al personal de su misma empresa, por lo que se recomienda cuidar los factores de presión sobre el evaluador.

En el proceso de evaluación se deben establecer reglas claras de las condiciones en las que se evaluará a un individuo. No es lo mismo ser sometido a evaluación en condiciones de total concentración a hacerlo mientras se realizan las funciones de trabajo.

El evaluador tiene un proceso de aprendizaje: aprende a planear el proceso de evaluación, a solicitar los materiales y equipo requeridos para las pruebas, a calcular el tiempo y el costo que le llevará aplicarlas, a cómo dirigirse a los candidatos que deben suspenderse para no provocar una reacción negativa, a cómo suspender a un candidato a quien ha encontrado haciendo trampa. Documentar las experiencias de los evaluadores ayuda a mejorar la práctica y a elaborar reglamentos para que puedan hacer mejor su trabajo.

$\mathrm{Al}$ analizar la relación que guarda la certificación de competencias laborales con el sistema de administración del capital humano en las dependencias de gobierno, se puede observar que aún no se ha integrado completamente la descripción de las normas al sistema. A pesar de eso, es obligatorio estar certificado para permanecer en la dependencia del gobierno federal, lo que seguirá reforzando al sistema de certificación en nuestro país.

Finalmente, consideramos que es necesario probar los beneficios económicos del modelo de certificación de competencia laboral para que sea un poderoso incentivo para atraer nuevos sectores y lograr el compromiso empresarial. La 
implementación y prueba de los beneficios económicos es fundamental, por lo que será necesario construir indicadores para el seguimiento del sistema y demostrar cuáles son las ganancias en productividad.

\section{Bibliografía}

Agudelo, Santiago, "Certificación ocupacional”, Manual didáctico, Cinterfor/OIT, Montevideo, 1993.

Baeza, Mónica y Mertens, Leonard, "La norma ISO 9000 y la Competencia Laboral", Consejo de Normalización y Certificación de Competencia Laboral, 2001 .

BID, "Programa Multifase de Formación de Recursos Humanos con Orientación de Competencias Fase 1", 2004.

CONOCER, "Análisis ocupacional y funcional del trabajo", IBERFOP- OEI, Madrid, 1998.

, "Estudio de Evaluación del Inicio de Operación del Sistema de Certificación de Competencia Laboral”, 2000.

, "La normalización y certificación de competencia laboral: Medio para incrementar la productividad de las empresas", Presentación en Power Point, Marzo, 1997.

1997.

, "Normalización y Certificación de Competencia Laboral", México,

, "Reglas Generales y Específicas de los Sistemas Normalizado y de Certificación de Competencia Laboral", Consejo de CONOCER, México, 1996.

, "Sistemas Normalizado y de Certificación de Competencia Laboral", México, 1997. 
, "Reunión anual de Evaluación del Proyecto de Modernización de la Educación Técnica y la Capacitación, Consejo de Normalización y Certificación de Competencia Laboral”, México, 1996.

Diario Oficial de la Federación, "Ley del Servicio Profesional de Carrera”, 10 de abril de 2003.

Dawkins, JS, “Skills for Australia”, Minister for Employmnet, Education andTraining, Canberra, 1987.

Galindo, Luis, "Técnicas de investigación en sociedad cultura y comunicación”, Prentice Hall, México, 2000.

Gonczi,Andrew, "Enfoques de la educación basada en competencias: la experiencia australiana", La academia, nov-dic, 1997.

http://www.hemerodigital.unam.mx/ANUIES/ipn/academia/12/sec_9.htm

Gonczi,Andrew, "Problemas asociados con la implantación de la educación basada en competencias: de lo atomístico a lo holístico", en Organización Internacional del Trabajo, Formación basada en competencia laboral, México, 1997.

Ibarra, Agustín, "El desarrollo de los sistemas normalizado y de certificación de competencia laboral y la transformación de la formación y la capacitación en México", Ponencia presentada en el Encuentro Andino de Formación Basada en Competencia Laboral, Bogotá, Mayo, 1998.

Jackson, Nancy, "Reforming vocational learning:Contradictions of competency", en What future for technical and vocational education and training, vol.2, NCVER, Adelaide, 1992.

Mertens, Leonard, "Competencia laboral: sistemas, surgimiento y modelos",OIT, Montevideo, 1996.

OIT, “Formación profesional. Glosario de términos escogidos”, Ginebra, 1993.

Pedrero, Fernando, periódico Reforma, 15 de julio de 2002. 
Kellie Deborah, "The australian way: competency based training in the corporate sector”, International Journal of training and development, v 3, no.2, jun, 1999.

Sierra, Francisco, "Función y sentido de la entrevista cualitativa en la investigación social", en Coord. Jesús Galindo, Técnicas de Investigación en Sociedad Cultura y Comunicación, México, Pearson Education, 1998.

Taylor, Catherine, "Assessment for measurement of standards: the peril and promise of large scale assessment reform", en American Educational Research Journal, vol. 31, no. 2, 1994, pp. 231-62.

\section{FUENTES DE INTERNET:}

http//: www.conocer.org.mx.

http:// www.presidencia.gob.mx

http://www.bancomundial.org.mx/pdf/3805-ME.pdf

http://www.gestiopolis.com/canales/derrhh/articulos/64/clicm.htm

http://www.iingen.unam.mx/areas/isi/abatm.html $\mathrm{g}$ 